ISSN: $1130-3743$

\title{
ANTECEDENTES DE LA CONSTRUCCIÓN TEÓRICA EN ANTROPOLOGÍA DE LA EDUCACIÓN
}

\author{
Antecedents of theoretical construction in the antrophology \\ of education
}

Petra M. PÉREZ ALONSO-GETA

Departamento de Teoría de la Educación. Facultad de Filosofía y Ciencias de la Educación. Universidad de Valencia. Avda. Blasco Ibáñez, 21. 46010 Valencia.

BIBLID [(1130-3743) 10, 1998, 205-231]

RESUMEN

El estudio sobre el hombre surge en el ámbito filosófico. La teoría de la evolución al situar lo humano en el ámbito de la naturaleza da un nuevo sentido al saber antropológico inaugurando las antropologías de corte empírico-positivas. En el campo de la antropología cultural, en las últimas décadas, con la desaparición del "exotismo" y la necesaria incorporación al estudio de las sociedades complejas, ha sufrido profundas transformaciones. En este sentido, si los datos antropológicos han de generalizarse a nivel global, deben combinar el uso de técnicas cualitativas y cuantitativas.

En el campo conocido como "Antropología y Educación" el uso de técnicas etnográficas para el estudio del aula y la escuela es bastante unánime, pero si sus datos han de servir a la toma de decisiones educativas de ámbito general es necesario avanzar por los caminos de los análisis antropológico-sociales, en las llamadas sociedades complejas.

\section{SUMMARY}

The study of mankind occurs within a philosophical environment. The theory of evolution places all chat is human in a natural environment and such gives a new meaning of anthropological knowledge by creativy anthropologies of an empirically positive nature. As far as social sciences are concerned, the Antropology has 
had important transformations in the last decades when the "exotism" disappeared and there has been a necessary study of the complex societies. In this way if the antropological data has to be generalised to a global level, the use of qualitative and quantitative tecniques has to be combined.

In the field known as "Antropology and Education" the use of Etnographic tecniques to study the school is used by most people, but if their information has to be used to take important decisions in Education, it's necessary to make progress in the antropological-social anaiisis, in the well-knowh complex societies.

\section{LOS SABERES ACERCA DEL HOMBRE: ANOTACIONES PREVIAS}

Las primeras interpretaciones de la naturaleza humana, nacen en el campo filosófico, en torno a la pregunta ¿qué es el hombre? Surge así la Antropología filosófica.

La respuesta acerca del hombre, desde la Antropología filosófica, pretende tener validez para todos los hombres, en todas las culturas, y se fundamenta en el deseo humano de poseer un saber "total"; el amor a la sabiduría, que es el primero en el tiempo y como el pensamiento permanecerá siempre vivo, pero que está lejos de satisfacer las exigencias de verdad propia de la comprensión del hombre en todas sus dimensiones. Desde el punto de vista metafísico, la noción del hombre queda perfectamente delimitada, dentro del género animal, por la racionalidad humana como diferenciación específica.

En el camino del saber antropológico un hito fundamental lo constituye la Teoría de la Evolución. Las investigaciones sobre el origen del hombre cobraron a partir de ahí un nuevo giro, el "evolucionismo" marca el comienzo de la Antropología científica, porque es la primera vez que se formula una teoría general de máximo alcance que permite construir hipótesis susceptibles de verificación empírica. La Antropología empírico-positiva se ha desarrollado en diversos campos científicos, con preguntas como ¿qué seres en la naturaleza son los hombres?, y ¿cómo son los hombres en su naturaleza biológica?, que delimitan el objeto de la Antropología física; o ¿cómo es el sistema sociocultural? y, ¿cómo funciona?, que describirían el estudio de la Antropología Social y Cultural. Este enfoque empíricopositivista se ha desarrollado también específicamente en otros campos científicos, dando lugar a la Antropología cognitiva, Antropología lingüística, Antropología política, etc. Dentro de la Antropología cultural y social surge la corriente cultura y personalidad antecedente inmediato de la Antropología de la Educación de corte científico.

\subsection{La Antropología Cultural y la corriente cultura y personalidad}

La Antropología Cultural se interesa muy pronto por el estudio de la educación y los procesos de transmisión cultural. Este interés se remonta a finales del 1800. Fue ya entonces cuando se constató por primera vez las contribuciones que la Antropología podría hacer a la educación y la comprensión de la cultura de la infancia (Barnes, 1896; Chamberlain, 1896; Fletcher, 1888; Stevenson, 1887; etc.). 
En esta misma línea se inscribe, el interés de F. Boas al enviar a Margaret Mead a estudiar el período de la adolescencia en la población primitiva de Samoa en 1928. En esta investigación Mead ya planteaba la necesidad de comparar la civilización norteamericana con otras sociedades "más simples", con fines prácticos, tendentes a solucionar los problemas educativos.

Tanto en Estados Unidos como en el Reino Unnido, lo que empezó como etnografías infantiles y estudios de los efectos de la cultura en los niños, se fue aplicando poco a poco a la solución de los problemas educativos, especialmente los que resultaban del contacto entre diferentes culturas. En Gran Bretaña, los programas se dirigían a intentar mejorar la educación en las colonias africanas. A un lado y otro del Atlántico, se asistía al nacimiento de la Antropología social y cultural moderna y el desarrollo intelectual de las mismas se veía influenciado significativamente por el crecimiento de un movimiento interdisciplinar en las ciencias sociales (Sociología, Antropología, Psicología, Pedagogía).

En la década de los 30 se consolida dentro de la Antropología cultural la corriente Cultura y Personalidad de orientación Boasiana, corriente clave que orienta el nacimiento de la Antropología de la Educación. En esta época, junto a los estudios de la adolescencia, interesan a F. Boas y sus discípulos (Mead, 1972 y Benedict, 1934) los primeros años de vida del niño y su configuración cultural, ya que a través de la crianza, entienden, se conforma la personalidad del individuo. Con estos estudios, la educación pasa a ser el eje fundamental de sus intereses, por el papel que juega en la confluencia de cultura y personalidad, (Colom y Janer, 1995, p. 84). Sin embargo, a nivel teórico, los antropólogos cuestionaban a Watson, Piaget y al propio Freud, pues éstos, olvidando las variaciones culturales, generalizaban acerca del desarrollo y el comportamiento humano, lo que suscitó un gran debate en cuanto a la concepción de la naturaleza intelectual del niño, con implicaciones importantes en la política educativa del momento. A su vez, en los Estados Unidos, la aplicación de las metodologías del trabajo de campo revelaron que dentro de la sociedad americana existía una diversidad de formas de enculturación y participación escolar, dependiendo de la etnicidad y clase social. En 1941 los antropólogos junto a profesionales de la Psicología y Medicina participaban en problemas de cooperación y acción social orientados a la recogi$\mathrm{da}$ de datos científicos que posteriormente sirvieran de base para preparar las recomendaciones y acciones, con el fin de mejorar las políticas federales respecto a los problemas educativos y administrativos de los Indios.

Una revisón bibliográfica sobre los estudios antropológicos llevados a cabo sobre la infancia y Educación revela que numerosos antropólogos se dedicaron a la investigación de los de sistemas formalizados de la educación y la enculturación del niño entre 1925 y 1954 . En el listado aparecen los nombres más representativos de la Antropología moderna Británica y Norteaméricana (Eddy, 1985, p. 88). En este momento la antropología ya había roto con las teorías de la evolución unilineal y la difusión extrema que la caracterizaron en el siglo XIX. Además, la publicación en Inglaterra en 1922 de la conocida obra, Los argonautas del Pacífico Oeste de Malinowski y la de Los Isleños de Andaman de Racliffe-Brown, anunciaba el comienzo de la Antropología práctica o aplicada. Se sostenía la estrecha rela- 
ción entre la Antropología teórica y aplicada, ambas orientadas al estudio del comportamiento humano y de las instituciones en el mundo contemporáneo. Desde esta base, se mantenía que los conocimientos antropológicos podían ser aplicados por los encargados de diseñar las políticas administrativas para la solución de los problemas educativos de las poblaciones nativas.

\section{Los Saberes ANTROPOlógicos-Educativos}

La clave de la visión antropológico-educativa del hombre, puede resolverse en una imagen representada por una intersección de círculos que convergen en una superposición interactiva de naturaleza bumana, educación y cultura.

Cuando se concibe la cultura como una serie de dispositivos simbólicos, como un sistema de significación creado históricamente en el tiempo por un grupo humano, en virtud del cual los individuos ordenan sus vidas de acuerdo con determinadas formas, esta cultura se define en el hombre en la medida en que suministra el vínculo entre lo que los hombres tienen posibilidad de llegar a ser y lo que realmente llegan a ser como miembros del grupo cultural. Desde esta perspectiva, la educación no es solamente proceso de transmisión cultural, sino también forma cultural de definición del hombre.

A partir de aquí surgen dos modelos de análisis antropológico-educativos que, en el marco de lo filosófico y lo científico, se preguntan sobre el qué y el cómo de la educación y por el qué y el cómo del hombre en su calidad de educable. Las perspectivas filosófica y científica versan sobre los mismos objetos, desde el punto de vista formal. La primera se pregunta por la esencia de éstos, la segunda, tal y como aquí se entiende, los describe, explica y "predice".

\subsection{Antropología Filosófica de la Educación}

$\mathrm{Si}$, como se ha dicho, es aconsejable caracterizar los saberes por sus métodos y no por sus resultados, las preguntas sobre el qué y el cómo de la educación y del hombre en calidad de educable se resuelven aquí en torno a la metodología filosófica, que diversificada a su vez en sus métodos, puede dar lugar a diversos enfoques en la definición de lo humano. Si bien es característico de la reflexión filosófica en educación que sus resultados puedan servir para cualquier tipo de hombre y en cualquier cultura, como un saber de "validez universal". Desde este enfoque se parte del supuesto de que el hombre es más de lo que las ciencias positivas pueden decir de él. En este sentido, la pregunta sobre qué es propiamente el hombre como "ens educandus" y cuál es el sentido de la educación constituye uno de los temas centrales de la perspectiva filosófica en Antropología de la Educación.

Otra de las prioridades, que entendemos fundamental, es la preocupación por la búsqueda de una imagen unitaria del hombre y la educación, una visión integral siempre en proceso de búsqueda, que se apoya en las aportaciones de las ciencias positivas que tienen que ver con el hombre y su educación. Una búsqueda que no acabará nunca, porque, en cuanto saber filosófico, los datos sobre 
los que reflexiona evolucionan con el conocimiento científico mismo. La misión de este saber es llegar a emitir "juicios de valor" en torno al deber ser de la educación, del hombre y la cultura, desde la perspectiva educativa. Pero también puede emitir juicios de verdad acerca de los contenidos científicos sobre los que se apoya, buscando la visión holista y unitaria de su objeto de estudio.

\subsection{La Antropología de la Educación como Antropología Científica}

En cuanto disciplina científica y, en tanto se entienda por "científico" lo que es propio de ciencias humanas, métodos cón tendencias a la objetividad, explicaciones probabilísticas, etc., nuestra propuesta es ambiciosa y quiere caminar desde la comprensión y descripción hacia la explicación, generalización y "predicción", que es la línea trazada para la ciencia y el camino que siguen las ciencias sociales.

Cualquier reflexión ha de partir de datos. Una reflexión sin datos es una reflexión ciega. Entendemos que, además de "otros" datos, se trabaje, como dice Geertz, sobre datos reales, de individuos reales, en culturas reales.

Señala Geertz que necesitamos:

"Una teoría de los valores que considere la conducta de la gente real en sociedades reales con culturas reales como su estímulo y su validación nos apartará de los argumentos abstractos"... "con los que vuelven a afirmarse una y otra vez unas pocas posiciones clásicas con muy poco de nuevo que las recomiende, y nos llevará a un conocimiento y comprensión cada vez mayor de lo que son los valores y de la forma en que funcionan. Una vez que esté bien orientada esta empresa del análisis científico de los valores, las discusiones filosóficas sobre la ética adquirirán probablemente más peso. No se trata aquí de reemplazar una filosofía moral, una ética descriptiva, sino que se trata de suministrar a la filosofía moral una base empírica y un marco conceptual que en cierto modo representen un avance sobre lo que nos ofrecen Aristóteles, Spinoza o G. E. Moore. El papel de una ciencia especial como la antropología en el análisis de los valores es no reemplazar la investigación filosófica, sino hacerla relevante» (Geertz, 1996, p. 130).

Geertz es un antropólogo empirista y resuelve su propuesta en clave de ciencia empírica. Sin embargo, nuestro proyecto antropológico es también educativo, y en educación hay que tomar decisiones de acuerdo a datos reales y, en muchos casos, de explicaciones probabilísticas científicamente contrastadas. Desde esta posición, que no pretende ser la única opción posible, debe entenderse, nuestra propuesta aquí, porque es en la ayuda a la toma de decisiones donde hoy se justifica cualquier ámbito científico.

Nuestra primera preocupación puede expresarse con la pregunta ¿de qué pretende ser ciencia la Antropología de la Educación?, en otras palabras ¿cuál es el objeto de la Antropología de la Educación? Dicha pregunta nos lleva directamente a consideraciones sobre el origen, alcance, desarrollo y estado actual de la disciplina. En esta línea podríamos hablar de la noción de "ciencia" y la demarcación de lo científico. Esta demarcación, si se adoptara un modelo clásico, nos llevaría 
a definir los criterios que se exigen para considerar una "ciencia" como los siguientes: cuantificación, verificación, simulación matemática y sistematización. Tras lo cual, en cuanto una investigación (sociológica, antropológica, pedagógica o psicológica) se aparta de este modo de proceder -cuantificador y sensorial- para seguir interpretaciones hermeneúticas, descripciones fenomenológicas, introspecciones, etc., no puede ser considerada como científica, sino como saber "filosófico", "metafísico" o incluso "literario".

Pero, de igual forma, se plantean otros criterios de demarcación de lo científico (Popper, Kuhn, Lakatos, Toulmin, Feyerabend). El racionalismo crítico de Popper, dirige las teorías científicas hacia una visión más "comprensiva" y para él, lo que constituye el rasgo distintivo del proceso científico es la crítica de conjeturas y el abandono de teorias refutadas. Como se sabe, el método para comprobar teorías no consiste para Popper sino en utilizar un criterio metodológico, el mayor grado de falsabilidad. La respuesta a Popper que obtuvo más eco fue, sin duda, la de Kuhn. Para él, la experiencia de falsación no sería una característica de la ciencia en períodos de normalidad (en los que se trabaja en los problemas que el paradigma implantado permite resolver), sino que la caracterizaría sólo en los períodos extraordinarios (en los que existen teorías en competencia, hasta que se impone un paradigma que proporciona nuevos modelos de problemas y soluciones a una comunidad científica).

Escapa a nuestro propósito hacer ni siquiera una pequeña síntesis de la historia de la filosofia de la ciencia o de los planteamientos sucesivos sobre el tema de Lakatos y Feyerabend, más aún, cuando la historia de la ciencia ha refutado a Popper en varios puntos. Entendemos con A. González (1978, p. 46) que Feyerabend es un relativista genuino y que si las teorías rivales son inconmensurables, según piensa Feyerabend, no hay ninguna razón para seguir sosteniendo que el desarrollo científico es racional. Popper, Feyerabend y la lógica inductiva actual tienen presupuestos metafísicos también. En este punto, la propuesta metodológica de Feyerabend es que proliferen todos los métodos y, lo mejor para el conocimiento, que compitan libremente todas las formas de conocimiento.

En lo que a nosotros respecta, la propuesta metodológica se resuelve intentando saber un poco más para actuar un poco mejor. Se concreta en un desarrollo del conocimiento que permite obtener "datos relevantes" de nuestro campo de estudio, sobre los cuales pueda apoyarse la toma de decisiones educativas. No en vano, en la ciencia actual, la aportación teórica a la "toma de decisiones" es un criterio básico de justificación de lo científico. Llegados a este punto seguimos siendo popperianos, en el sentido de que pensamos que cualquiera que sea nuestra Weltanschauung, nuestra visión del mundo y nuestra orientación teórica, las teorías que propongamos deben de ser tales que se puedan poner a prueba si quieren ser científicas.

\subsubsection{Sobre el carácter del estudio de los hechos sociales}

Augusto Compte (1929) fue el primero en utilizar el término sociología. Compte desarrolló lo que en un principio denominó "física social" y que más tarde 
acuñó como sociología dentro de su perspectiva científica: el positivismo o filosofía positiva. El uso del término física social ya evidenciaba el interés del autor por modular la sociología dentro de las ciencias más duras. Entendía que su estudio debía ser científico, al igual que la mayoría de los teóricos clásicos y de los sociólogos contemporáneos.

No vamos a hacer aquí una revisión en el tiempo de las teorías sociológicas, ni a reproducir descripciones precisas de las perspectivas metodológicas de determinados autores o grupos de científicos sociales, sino, siguiendo a los etnográfos Hammersley y Atkinson (1994), identificar tan sólo las corrientes de pensamiento paradigmáticas sobre la naturaleza de las ciencias sociales y sus formas de investigación, las cuales servirán de referencia a nuestra posición en torno a la investigación en Antropología de la Educación.

\section{El positivismo}

El positivismo ha tenido una larga historia en el pensamiento. Es característico del positivismo la aspiración a resolver los problemas humanos y sociales en términos de razón positiva. Esta razón positiva implica la subordinación de la imaginación a la observación. Hay una primacía de lo pragmático y de lo observable (Choza, 1985, p. 170). El positivismo alcanza su apogeo con el Círculo de Viena y ha tenido una notable influencia sobre los científicos sociales y en particular sobre los partidarios de la investigación experimental, de encuestas y formas cuantitativas de análisis asociadas a éstas.

El positivismo, como corriente de pensamiento que aspiraba al conocimiento científico de la realidad, ha ayudado considerablemente a la independencia epistemológica respecto a la Filosofía y también a otras ciencias sociales, como la Psicología o la Pedagogía (Colom y Manila, 1995). Los principales postulados del positivismo pueden ser formulados de la forma siguiente: a) El modelo de ciencia natural entendida en términos de la lógica de la experimentación es el modelo usado para la investigación social. b) El tipo de concepción científica adoptado es la explicación. A partir de las relaciones que se establecen entre las variables, se explican los eventos y se establecen las leyes de carácter universal. En la versión estadística de este modelo, las relaciones entre las variables tienen sólo una determinada probabilidad de aplicarse a todas las circunstancias. Desde esta perspectiva, cobran especial interés los procedimientos de muestreo. Esta técnica es precisamente importante por su capacidad de generalizar resultados. c) La observación neutral, estandarizando los procedimientos de observación. Se trata de elaborar criterios sólidos que sean estables para todos los observadores y conseguir así una base teóricamente neutra para trabajar. La característica más importante de las teorías científicas es que estén abiertas y sujetas a comprobación: confirmadas o refutadas. Es decir, lo que la teoría dice que ocurre en determinadas circunstancias ha de ser verificado. Para ello hay que fijar muy bien las condiciones y procedimientos de extracción de la información (orden y enunciados de las preguntas, condiciones de pase de los cuestionarios, etc.), de forma que pueda ser susceptible de comprobación. 


\section{El naturalismo}

Frente al positivismo, surge una perspectiva alternativa a la naturaleza propia de la investigación en ciencias sociales, que algunos han dado en llamar naturalismo, (Hammersley y Atkinson, 1994, p. 20). El naturalismo propone que, en lo posible, el mundo social debe ser estudiado en su estado natural, sin ser contaminado por el investigador, el cual ha de adoptar una actitud de respeto y fidelidad.

El naturalismo se mueve dentro de una amplia gama de corrientes, como la fenomenología, el interaccionismo simbólico, la hermeneútica, la etnometodología. Desde diferentes perspectivas, estos enfoques metodológicos coinciden en afirmar que los eventos sociales no pueden ser encasillados bajo según qué leyes, ya que en las acciones e interacciones humanas hay motivos, actitudes, creencias, "significados" sociales, que antedecen a la acción, y el uso de una metodología estandarizada no asegura la conmensurabilidad de la información. No es posible - para el naturalismo- comprender el comportamiento de la gente sin conocer el significado que guía ese comportamiento. Esto es mucho más obvio en el caso de culturas y grupos sociales diferentes a los nuestros, en los que no es posible ningún análisis si no se sabe interpretar el mundo de la misma forma que ellos. La propuesta no es la lógica de la explicación sino de la comprensión.

No vamos a entrar ahora en los desarrollos, problemas y sesgos metodológicos de los distintos supuestos y corrientes, sólo señalar, que se avanza hacia la superación de los que en ambos paradigmas pueden aparecer, así como hacia la combinación de las distintas metodologías, sin olvidar el objetivo específico de la investigación. En palabras de Hammersley y Atkinson: "Para el positivismo, el objetivo es descubrir leyes universales o al menos elaborar explicaciones acerca de fenómenos particulares formuladas en términos de leyes universales. Para el naturalismo la única tarea legítima es la descripción cultural"... "Desde nuestro punto de vista, la función distintiva de la teoría en ciencias sociales es el desarrollo y verificación de teorías; esto es lo que la diferencia del periodismo y de la literatura, aunque tenga cosas en común" (1994, p. 34).

Si bien brevemente, en este punto hay que hacer referencia al menos a la corriente sociológica americana de la etnometodología. Nace en la Universidad de California en los años 60 y supone una ruptura radical con las formas de pensamiento de la sociología tradicional. Se caracteriza por un enfoque cualitativo en la investigación del mundo social en contra del paradigma cuantificador dominante. Siguiendo a Alfred Schuzt y Parsons, se entiende que la realidad ya está descrita por la gente. El lenguaje ordinario expresa la realidad social, la describe y la construye al mismo tiempo. La etnometodología se ocupa también de la intervención social, entendiendo que todo grupo social es capaz de comprenderse a sí mismo, comentarse y analizarse. La etnometodología tiene puntos comunes con el marxismo, existencialismo, la fenomenología y el análisis institucional. De ahí el interés que puede ofrecer para algunos enfoques de la Antropología en general (Antropología crítica) y de la Antropología de la Educación en particular. 


\section{Aportaciones teóricas: hacia un paradigma en ciencias sociales más integrado}

Por otra parte, desde la construcción teórica en ciencias sociales, hoy, se percibe la necesidad, partiendo de la naturaleza multiparadigmática contemporánea de las ciencias sociales, de avanzar hacia un paradigma sociológico más integrado, ya que muchas veces las diferentes propuestas son perceptivamente diferentes, pero mutuamente enriquecedoras. Así, los conductistas sociales se esfuerzan por integrar los procesos mentales creativos (elemento central de las definiciones sociales del interaccionismo simbólico, fenomenología, etnometodología) con el conductismo tradicional (Ritzer, 1993).

El mundo social es muy complejo y, para poder comprenderlo, se requieren modelos relativamente simples. Ante la demanda de un paradigma integrado, Ritzer afirma textualmente: "el modelo simple que estamos buscando se deriva de la intersección de los dos continua de los niveles de la realidad social"... "el primero, el continuum microscópico-macroscópico" (Ritzer, 1993, p. 609). El segundo es el continuum objetivo-subjetivo. Entre los objetivos -que tienen una existencia material y es posible verlos y tocarlos- tenemos entre otros los actores, la interacción, etc. Los subjetivos, sin embargo, existen sólo en el mundo de las ideas, carecen de existencia material. Se trata de fenómenos tales como los procesos mentales, (Berger y Luckman, 1968), las normas, los valores y muchos otros elementos de la cultura.

\subsubsection{Sobre el "carácter científico del estudio antropológico"}

Al pretender un tratamiento científico para la Antropología de la Educación somos conscientes de que estamos defendiendo una "opción determinada", que exige, como paso previo, ocuparse de las objeciones fundamentales que se han hecho a la utilización "unitaria" del método científico. Las objeciones suelen referirse a la extensión del "método científico", a las ciencias sociales en general y a la Antropología en particular. Se basan en la complejidad, bolismo y subjetividad de los hechos sociales. La consecuencia es que éstos se caracterizan por algunas notas que dificultan la aplicación del método científico a este ámbito del saber.

A la objeción de complejidad ya respondió Popper en los años cuarenta, argumentando que si bien es verdad que el análisis de cualquier situación social concreta se hace difícil por su complejidad, lo mismo ocurre con cualquier situación física determinada. La tesis de imposibilidad de tratamiento científico es aplicable también a los fenómenos físicos (Popper, 1995a, p. 371). En cuanto a la naturaleza bolística de los hechos sociales, la idea que subyace es que el grupo social es más que la mera suma total de sus miembros y que los grupos nunca pueden ser considerados como meros agregados de personas. Y si bien es verdad que el tratamiento holístico, como ideal, es incompatible con el método científico, hay que responder, siguiendo a Nagel (1978, p. 403), que, si esto es cierto en las ciencias sociales, lo es también para todas las ciencias que estudian grupos vivientes (ciencias biológicas). Ciertamente, en antropología se han hecho con frecuencia profesiones de fe holística, sin embargo, hay que responder con 
A. González que "como ideal está en desuso", (1987, p. 206). Los desarrollos históricos (avance de la industrialización, de los mass media, idea de la aldea global, etc.) de los últimos años han obligado a los antropólogos a atemperar su forma de aproximación holística (González, 1987).

En cuanto al sesgo "subjetivista" (siempre impregnado de valores) que se introduce por parte del investigador al estudiar los hechos sociales, por su significación para la investigación en Antropología de la Educación, intentaremos abordarlo más adelante en otro trabajo posterior.

\section{El estudio antropológico en las sociedades complejas}

Antes de entrar en el ámbito de estudio que nos es propio hay que considerar, si bien brevemente, el desplazamiento del campo de actuación que la antropología, con carácter general, ha experimentado en los últimos años. Ante la drástica desaparición del llamado "exotismo" de grupos humanos cerrados en sí mismos, en los que era posible el estudio de unidades a pequeña escala y la observación participante como trabajo de campo, algunos antropólogos han reaccionado buscando las pocas sociedades susceptibles todavía de este tipo de estudios (Nueva Guinea y algunas partes de Sudamérica). Otros no han tenido más remedio que desplazar el campo de estudio de estas entidades al de "unidades" como una aldea, un hospital, un gueto, una escuela, etc., dentro de las sociedades complejas (sociedades preindustriales e industriales). Sin embargo hay quienes han dudado del valor de ocuparse de estas situaciones de pequeña escala en las sociedades complejas, ya que no pueden considerarse autónomas. Al hacerlo así, aseguran, se pueden perder las relaciones y estructuras más significativas que definen y gobiernan la sociedad. Tales estructuras trascienden al grupo específico de estudio y no se prestan fácilmente a la metodología de trabajo de campo antropológico tradicional.

Para este mismo grupo de antropólogos, el hecho de limitar el estudio antropológico a aquello que puede ser manejado por los métodos tradicionales del trabajo de campo constriñe innecesariamente la disciplina. Piensan que la Antropología debe trasladar su campo más allá de los barrios, aldeas, etc., esto es, a los análisis "holísticos" de los sistemas a gran escala, como las nacionesestado (Kaplan y Manners, 1988). Se trata de recuperar el holismo, la forma de aproximación comparativa y holística, que consideran el rasgo más fructífero del método antropológico, en detrimento de la técnica de observación participante.

Otros antropólogos han señalado la dificultad que conlleva hacer estudios holísticos a gran escala, ya que si bien están de acuerdo en que no es posible seguir suponiendo que la mayor parte de las unidades sociales de pequeña escala son autónomas, creen que no es lo mismo tratar de forma holística los "zuñi" o los "nuer" de la China que la India Moderna (Kaplan y Manners, 1988). Para complicar más las cosas acerca de la indefinición del campo antropológico, desde otra perspectiva, el antropólogo Geertz afirma recientemente, refiriéndose a la Antropología que: 
"No llega a definirse ni por el método ni por el objeto de estudio, aunque frecuentemente en relación al primero se usa el término "etnografía" y en relación al último el de "sociedad primitiva". Pero la primera oscurece tanto como se supone que clarifica y la segunda está mal concebida"... "La Antropología o al menos la Antropología social y cultural, se asemeja más a algo que uno aprende mientras año tras año continúa intentando adivinar qué es y cómo se práctica, que a algo que se inculca a uno por medio de algún "método sistemático" (1996a, pp. 101-102)".

\section{La propuesta de Geertz para la Antropología es que:}

"Creyendo con Max Weber que el hombre es un animal inserto en tramas de significación que él mismo ha tejido, considera que la cultura es esa urdimbre y que el análisis de la cultura ha de ser por lo tanto, no una ciencia experimental en busca de leyes sino una ciencia interpretativa en busca de significaciones. Lo que busco es la explicación interpretando expresiones sociales que son enigmáticas en su superficie" (1996b, p. 20).

Para Sánchez, sin embargo, las concepciones de Geertz acerca del hombre y la cultura junto a su "crítica del ideal explicacionista que la Antropología ha tenido en cuanto disciplina empírica de pretensión científica, le abocan a un particularismo de consecuencias relativistas» (Sánchez, 1996, p. 26). Puestas así las cosas, nuestra propuesta sigue siendo apostar una vez más por una Antropología científica de la educación, que, parafraseando a Lévi-Strauss, nos permita saber un poco más para poder actuar un poco mejor.

Después de constatar (Beattie, 1988, p. 293) que los antropólogos "no han estado siempre de acuerdo sobre la índole exacta ni de su objeto de estudio, ni del tipo de interés que tiene por él", no nos queda sino acercarnos a ver qué estudia actualmente la Antropología y qué sentido se da a lo que estudia. Si tratamos de ver qué es lo que hacen los antropólogos, para empezar, hay que señalar que no se limitan a los pueblos "primitivos" sin escritura o exóticos, sino que hoy investigan comúnmente en sociedades europeas, americanas o asiáticas, que no son en ningún sentido primitivas. Son las llamadas sociedades complejas. En segundo lugar, como señala el mismo Beattie (1988):

"Los antropólogos sociales no estudian ni comparan sociedades "enteras", si se entiende por "sociedad" (como es común) un tipo u otro de totalidad empírica, tal cosa sería imposible. Lo que hacen o, por lo menos, una de las cosas que hacen, es abstraer del comportamiento social que observan determinados aspectos o características constantes o institucionalizadas que parecen ir unidas y tener sentido según los términos de algún interés especial del observador"... "De modo que el objeto de la antropología social o, por lo menos, de una parte importante de ella es descrito más exactamente como las relaciones sociales institucionalizadas y los sistemas en que éstas se ordenen, más que como "la sociedad" o "las sociedades", consideradas como totalidades que de alguna manera se ofrecían al observador como entidades empíricas" (p. 294).

También Popper (1995b), en torno al peligro del enfoque "holístico" en las ciencias sociales, señala que "el pensamiento holístico es en especial nefasto"... "Si 
queremos estudiar algo, estamos obligados a seleccionar aspectos determinados de ello. No nos es posible observar ni describir un pedazo entero del mundo o un pedazo entero de la naturaleza... "puesto que cualquier descripción es necesariamente selectiva" (p. 332).

Los antropólogos estudian lo que hace la gente y lo que piensa acerca de lo que hace en una determinada cultura, a través de técnicas cualitativas, fundamentalmente etnográficas y a veces cuantitativas. Sin embargo, acerca de lo que se hace, la gente desarrolla ideas de dos tipos: primero, sus nociones sobre lo que de hecho hacen y segundo, sus creencias sobre lo que debieran hacer (normas o valores éticos); expresado en palabras de Beattie (1988):

"Puede decirse que los antropólogos sociales en realidad se ocupan de tres tipos o niveles de datos distintos; I) "lo que ocurre de hecho", II) lo que la gente piensa que ocurre, y III) lo que piensa que debiera de ocurrir, es decir, sus valores legales y morales"... "lo que de hecho ocurre" puede a menudo ser tratado cuantitativamente "... "con los otros dos tipos, las creencias y los valores"... "hay que advertir que incluso una afirmación estadística exige, si pretende tener sentido, una definición cualitativa de sus términos; la información citada anteriormente es sólo informativa sociológicamente si se sabe lo que significan dentro del contexto social y cultural en que se realiza la investigación" (p. 296).

En cuanto a cómo son empleados en los informes los datos extraídos en la investigación antropológica, hay que decir que la tarea más inmediata es la descripción. Sin embargo, las descripciones hacen algo más que estrictamente describir, si entendemos que toda descripción se hace en términos generales y que los términos generales son abstracciones que se hacen sobre los datos. Consecuentemente las descripciones en cierta forma son explicativas; más aún si entendemos con Nadel (1974, p. 61) que: "La explicación siempre está en el campo de la descripción". La explicación da sentido a lo que de por sí solo es ininteligible. Los hechos cobran valor en cuanto se ven como parte o como ejemplificación de un sistema o de un proceso (Beattie, 1988). En términos generales la explicación, en Antropología, ha permitido relacionar los datos como un sistema de acontecimientos y cosas, dentro de un marco general comprensivo, de tal forma, que permite dar una visión global de los hechos, de manera que ya no se perciben como datos aislados sino ensamblados en un esquema común.

Ahora bien, en Antropología, junto a la descripción y la explicación, hoy es también posible la predicción. La propuesta de predicción aquí, puede entenderse en sus justos términos siguiendo a Nagel (1978): "Si el conocimiento que los hombres poseen de los procesos sociales es una variable que interviene en la determinación de los fenómenos sociales, no hay ningún fundamento a priori para sostener que los cambios de esta variable y los efectos que ellos puedan producir no puedan ser objeto de leyes sociales" (p. 425). Por ejemplo, las predicciones que pueden hacerse después de un sondeo, en las que, sin duda, además de la descripción y la explicación es posible algún tipo de predicción.

Por otra parte, este tipo de estudios es adecuado para la investigación en las llamadas sociedades complejas. En palabras del antropólogo Kottak (1996): 
"Una combinación de investigación con encuestas y de etnografía puede proporcionar nuevas perspectivas sobre la vida de las sociedades complejas (sociedades grandes y populosas con estratificación social y gobiernos centralizados). Una etnografía preliminar puede ayudar también a desarrollar preguntas relevantes y culturalmente apropiadas para su inclusión en encuestas nacionales" (p. 19).

Otra cuestión será la del alcance que permitan las predicciones en Antropología, sin duda, siempre probabilístico. En este sentido, cabe mencionar que el mayor alcance corresponde a la Antropología aplicada, que permite poner a prueba las predicciones a través de los llamados "experimentos sociales fragmentarios".

En resumen, a través de la observación, los antropólogos estudian --en términos de interacción sistemática- el comportamiento de la gente y las relaciones que establecen. Pero se ocupan también de los valores y creencias que subyacen a tales comportamientos y relaciones. Sus informes han tenido tradicionalmente un carácter descriptivo y explicativo y han intentado dar una visión comprensiva de las sociedades y culturas. La comprensión, algo por lo que los antropólogos han estado especialmente interesados, se consigue precisamente a través del conocimiento de las creencias y valores de la gente que se estudia. Para ello, es imprescindible "vivir" en la cultura, conocer sus creencias, valores y estilos de vida (lo mental y lo conductual), única forma de acceder a su sistema de significaciones.

Pero si hemos de atender también a lo que actualmente se hace en Antropología (o al menos en una parte importante de los estudios), hay que señalar que se avanza cada vez más desde la Descripción y Explicación a la Predicción. Desde esta perspectiva, la investigación antropológica para el estudio de las llamadas sociedades complejas utiliza una combinación de las técnicas cualitativas clásicas (etnografía), con técnicas cuantitativas, que a nivel probabilístico permiten generalizar y fijar las condiciones de aparición y construcción de eventos sociales. Es decir, la Predicción.

\subsubsection{La investigación en Antropología de la Educación}

Siguiendo nuevamente a Kuhn, el concepto clave que aquí nos interesa es el de paradigma; si bien es verdad que Kuhn empleó el término en varios sentidos, vamos a utilizar, en lo que a nosotros interesa, la formulación de Ritzer, que entendemos fiel al significado global de la obra de Kuhn:

"Un paradigma es una imagen básica del objeto de una ciencia. Sirve para definir lo que debe estudiarse, las preguntas que es necesario responder, cómo deben responderse y qué reglas es preciso seguir para interpretar las respuestas obtenidas. El paradigma es la unidad más general de consenso dentro de una ciencia y sirve para diferenciar una comunidad científica (o subcomunidad) de otra. Subsume, define e interrelaciona los ejemplares, las teorías y los métodos e instrumentos disponibles" (Ritzer, 1975, p. 70).

Con esta definición podemos delimitar varios conceptos que nos interesan para identificar los paradigmas fundamentales de la Antropología de la Educación, 
ya que la explicitación de las perspectivas paradigmáticas y teóricas son fundamentales, pues a partir de ellas se resuelven los resultados de la investigación antropológica. Las teorías serían sólo parte integrante de paradigmas más amplios, ya que éstos pueden abarcar varias teorías, así como diferentes formas de entender el objeto de estudio, los métodos (e instrumentos) y las líneas específicas de trabajo científico, que constituyen modelos o ejemplificaciones para los que las siguen.

Los métodos de investigación y las opciones metodológicas son parte integral de un marco o sistema teórico más amplio empleado por el investigador. Si bien cada investigador debe explicitar el marco teórico de su trabajo, hay que constatar que no es frecuente encontrar en las investigaciones en Antropología de la Educación referencia a los diseños o sus marcos teóricos, ni tratamientos explícitos que relacionen paradigma, teoría y resultados de la investigación antropológico educativa. En este sentido, numerosas críticas se han vertido acerca de los supuestos teóricos y metodológicos que desarrollan las investigaciones etnográficas y no etnográficas en Antropología de la Educación (Tindall, 1976; Smith, 1974; Eisenhart, 1988; Geertz, 1996b; etc.). No obstante, el análisis de la relación teoríadiseño no se menciona o aparece como periférico (Sindell, 1974; Goetz, 1978; etc.). Aún es más difícil encontrar investigaciones donde aparezca la teoría como marco conceptual del que parte el investigador y como elemento integrante del diseño y elaboración de los informes.

La Antropología de la Educación, aunque desde los años sesenta y setenta vive una época floreciente, haciendo una revisión de la investigación, se observa que todavía no constituye un área de investigación bien definida. A pesar de ello, representa un enfoque antropológico de los procesos y problemas educativos. En palabras de Goetz y Le Compte: "En esencia, constituye una síntesis interdisciplinar emergente, al practicarla investigadores de distintas tradiciones. Sin embargo, éstos no han llegado a un consenso respecto a cuál debe ser su alcance y su método" (1988, pp. 42-43). Dentro del campo de la Antropología de la Educación, aún sin explicitar, se han utilizado tradicionalmente marcos teóricos del ámbito de las ciencias sociales -Sociología y Psicología- fundamentalmente. Los etnógrafos no comparten una única definición de cultura y en sus trabajos han sido influenciados por otras disciplinas (Psicología Educativa, Medicina, Sociología de la Educación, etc.) y por orientaciones positivistas. Por eso, sus aproximaciones metodológicas específicas varían considerablemente (Jacob, 1987). Además, en el campo de la educación, especialmente a partir de la década de los ochenta, se han desarrollado estudios etnográficos que son virtualmente idénticos a los que llevan a cabo los antropólogos (Goetz y Le Compte, 1988) alentados por una práctica que conecta con toda una filosofía de investigación educativa -en la década de los ochenta-dominante: la idea del "profesor investigador" que debe adoptar una postura de indagación sobre su práctica.

Comparando la evolución de los estudios etnográficos con el cambio de los paradigmas teóricos (en las ciencias naturales) de Kuhn, se observa la influencia de las perspectivas teóricas dominantes en ciencias sociales y cómo las perspectivas disciplinares se desarrollan, orientan las investigaciones y son, finalmente, sus- 
tituidas por otras perspectivas emergentes. Ello indica que, entre los etnógrafos del área de la educación, las teorías Estructural-Funcionaly del Conflicto fueron sustituidas progresivamente por paradigmas, tales como la Teoría de Sistemas y, más recientemente, el Interaccionismo Simbólico y la Teoría del Intercambio Social. Otras veces, la investigación de determinados temas forzó sobre la marcha el cambio del marco teórico. Éste es el caso de la investigación de Smith y Geoffrey sobre la "interacción de profesores y alumnos en el aula", que se desarrolló partiendo de un problema de la experiencia docente de Geoffrey y bajo el marco teórico del Estructural-Funcionalismo, el Conductismo y la Teoría Psicológica de la Personalidad. Los constructos que fueron apareciendo obligaron a una revisión de las cuestiones iniciales y a nuevos análisis de los datos. Como consecuencia, al final del estudio los investigadores habían incorporado aspectos relevantes de varias teorias alternativas (Smith y Geoffrey, 1968).

Por otra parte, no hay que olvidar que aun contando con la influencia que el marco teórico tiene en la selección de estrategias para la recogida de datos, se observa en las ciencias sociales una tendencia ascendente a la utilización ecléctica de metodologías. En el caso del Paradigma de los Hechos Sociales (incluye a los teóricos del funcionalismo estructural y del conflicto, como dominantes, y la teoría de sistemas) suele utilizarse el método del cuestionario-entrevista. En el paradigma de la Definición Social (teoría de la acción, interaccionismo simbólico, etnometodología, etc.), el método de la observación es definidor de los partidarios de este paradigma, muchos de los cuales utilizan también el método del cuestionario-entrevista (Ritzer, 1993, p. 603).

\subsubsection{Explicación, contrastación y predicción en Antropología de la Educación}

\section{La explicación científica}

La definición de explicación, desde un punto de vista gnoseológico, debe hacer referencia directa al conocimiento. En este sentido, las explicaciones, según Nagel, "pueden ser consideradas como intentos de comprender lo no conocido, en términos de lo conocido a algo más conocido" (1978, p. 109). El fin de toda explicación, desde esta perspectiva, no puede ser otro que el hacer comprensible o más comprensible algo. Las explicaciones científicas tratan de dar explicaciones no de un acontecimiento o suceso singular, sino de todo un conjunto de ellos. Son explicaciones de tipo general, se basan en la formulación de leyes.

Dentro de las "explicaciones científicas de acontecimientos", cabe destacar las "explicaciones de las acciones", que, aun dentro de éstas, tienen la particularidad de que en ellas interviene la libertad humana, las intenciones, propósitos y motivos del hombre. Por tanto, el carácter que se podía llamar "mecánico" de las explicaciones de sucesos puros, en las ciencias puras, resulta modificado o alterado por la imprevisibilidad propia de la libertad humana. Estas explicaciones son las peculiares de las ciencias humanas y sociales. En las ciencias en general, pero fundamentalmente en las ciencias sociales, no se tiene la seguridad de que muchos de los fenómenos explicados estén contenidos en la regla general, sino sólo probablemente. Por eso, las explicaciones en las ciencias sociales son probabilisticas. 
Las explicaciones son científicas no sólo porque utilizan leyes generales, sino y, además, en sentido estricto, porque la respuesta al porqué de la cuestión que hay que explicar es el resultado de una investigación científica. Un tipo de explicaciones que se pueden considerar como intermedias entre las científicas y no científicas son las explicaciones o interpretaciones "post factum". Según Merton, ocurren con frecuencia en la investigación social empírica. Consisten en que "se recogen los datos y sólo más tarde se someten a comentario interpretativo" (Merton, 1970, p. 103). Se caracterizan, pues, por la formulación de una interpretación después de haber hecho las observaciones, y no por la "comprobación empírica" de una hipótesis previamente formulada. La explicación científica tiene una importancia capital en el desarrollo científico. Como escribe Nagel «es el deseo de hallar explicaciones sistemáticas y controladas por elementos de juicio fácticos lo que da origen a la ciencia y es la organización y clasificación del conocimiento sobre la base de principios explicativos lo que constituye el objetivo científico de las ciencias" (1978, p. 17).

Desde este punto de vista, nosotros entendemos que la Antropología de la Educación debe avanzar hacia lo que es terreno común hoy en ciencias sociales, la explicación científica de carácter probabilístico, en la que las hipótesis puedan ser contrastadas.

\section{Contrastación y predicción en el trabajo de campo}

En los trabajos antropológicos, cada vez se es más consciente de la necesidad de que la observación esté controlada por un diseño que especifique los datos relevantes y permita poner a prueba generalizaciones relativas a las relaciones entre variables (Levine, 1970; Hammersley y Atkinson, 1994; González, 1987; etc.). En este sentido, los parámetros descriptivos, comparativos y teóricos de la vieja Antropología van siendo sustituidos por teorías del trabajo de campo, por teorías del diseño experimental y por teorías sobre los tipos de datos que pueden refutar o corroborar las teorías antropológicas (González, 1978). Se trataría de desarrollar una nueva "jerarquía de teorías", de acuerdo con Suppes (1979), "media" entre las teorías -aquí antropológicas- y los datos.

A este nivel de desarrollo científico se resuelven los trabajos de la nueva etnografía, que permiten poner a prueba las hipótesis y ayudar al desarrollo de las teorías, sobre la base de un trabajo de campo realizado de acuerdo con un diseño previo. Desde esta perspectiva y cualquiera que sea la orientación teórica de partida, los límites del dominio de una hipótesis se proponen con carácter tentativo, aunque teóricamente fundados y se deben contrastar con los métodos apropiados. Ahora bien, cuando el trabajo antropológico estudia culturas no conocidas o aspectos de la propia cultura que desconocemos o de los que tenemos una visión "folk", las hipótesis sólo pueden llegar a formularse mediante un trabajo de campo exploratorio, o incluso después de él. En esta fase exploratoria o prospectiva, la recogida de datos debe hacerse de acuerdo con el background teórico previo, dentro del marco referencial y teórico existente. Ello exige la realización de trabajos de campo especialmente diseñados con este fin. En cualquier caso, ya se 
trate de un estudio prospectivo de generalización de bipótesis o ideado especialmente hacia la contrastación, para que el trabajo de campo resulte útil es necesaria la validación de conclusiones de acuerdo con un diseño previo, lo que requiere con frecuencia el uso de métodos estadísticos.

Cuando se piensa en el uso de métodos estadísticos en Antropología cultural se tiende a tener como referencia la utilización de datos etnográficos procedentes de un gran número de sociedades, bien sea para la puesta a prueba de hipótesis teóricas o para el establecimiento de comparación y relaciones entre culturas o de rasgos culturales en áreas de difusión (Howard, 1995, por citar sólo una investigación reciente). Pero en Antropología hay otros usos de métodos estadísticos y es cuando en el trabajo de campo se trata de establecer, en ámbitos más amplios, la corrección y el grado de generalidad de las hipótesis con las que se concluye la observación participante. La utilidad y necesidad de técnicas estadísticas se plantea siempre que el universo observado no coincide con la unidad de análisis. De forma que, en estos casos, resulta imprescindible precisar tanto la unidad de análisis como su vinculación con la contrastación de las conclusiones. No en vano los factores que se incluyen en una teoría antropológica explicativa delimitan si se precisa su campo de aplicación. Desde nuestra propuesta se entiende que la Antropología de la Educación ha de desarrollarse científicamente construyendo teorías sobre hipótesis que puedan contrastarse, mejorarse o rechazarse. Para ello es necesario que esas teorías puedan ponerse a prueba, ya que, entendemos, que el tipo de proposiciones que incluyen generalmente las teorías en Antropología de la Educación en las sociedades complejas son susceptibles de contrastación. Como también creemos que desde el posible ámbito de aplicación a que se dirigen se justifica esta u otra toma de posición.

En cuanto a la predicción en Antropología de la Educación, éste es un tema que viene preocupando desde lejos. Ya Brameld, uno de los padres del campo "antropología y educación" que se ocupó de la misma vinculándola a la causalidad, entiende:

"Que si hemos de comprender los procesos culturales no podemos evitar la indagación de las causas que originan el cambio cultural lo cual nos lleva, por supuesto, a la cuestión más fundamental en la filosofía de la ciencia, de qué se entiende por "causa" y su concepto gemelo, "efecto". De igual importancia es "predicción": ¿podemos, por un análisis cuidadoso del orden y proceso de la cultura, predecir un curso dado de acontecimientos culturales? La estrecha relación de esta cuestión con otras -por ejemplo, si podemos controlar deliberadamente un cambio cultural en direcciones deseables- es de principalísima importancia para la educación cuando se concibe como uno de los medios para tal control" (Brameld, 1961, p. 180).

El filosófo de la ciencia que quizás (hace ya dos siglos) propuso los supuestos de la teoría científica contemporánea relativa a la causalidad es David Hume. Para él, la causalidad se convierte en un concepto que connota, ya, las relaciones observables empíricamente entre sucesos concomitantes. A partir de Hume la complejidad inherente a la determinación de dichas relaciones ha conducido a 
trabajar cada vez más a partir de su probabilidad que de su certeza. Esto es todavía más concluyente en las ciencias sociales, donde la estadística y los métodos de establecer la probabilidad aportan grandes posibilidades de análisis.

Escribe Brameld que:

"Parece haber acuerdo entre muchos de los antropológos de nuestros días en que ninguna teoría del cambio cultural es atribuible a una sola causa -ni aun, que un conjunto límitado de causas específicamente definidas- es suficiente para explicar los intrincados procesos involucrados"... "Se supone que si la causalidad es tratada tan flexible y prudentemente, también debería serlo la predicción. Aparentemente predomina un consenso mayoritario de que el estudio científico de la cultura puede producir predicciones de tanteo, por lo tanto sólo probables, del curso del cambio cultural"... "Con tales limitaciones, sin embargo, la causalidad y la predicción continuan jugando un papel en la teoría de la cultura" (1961, p. 181). Para este mismo autor "si se consideran las "leyes culturales" como "enunciaciones de procesos", entonces no hay razón para que no sea lícito un cierto grado de predicción en lo tocante a las culturas particulares o las fases de cultura" (1961, p. 188).

La predicción en las ciencias sociales, actualmente, comprende dos tipos de métodos: cualitativos y cuantitativos. Los cualitativos se emplean cuando no se parte de información anterior sobre el fenómeno considerado. En los métodos cuantitativos se extrae toda la información posible contenida en los datos y, en virtud de los patrones de conducta observados en el pasado, se realizan las conjeturas y predicciones sobre el futuro (Uriel, 1995, p. 26).

Ya en el campo de la etnografía escolar, Erickson señala también la posibilidad de explicación causal y la predicción en la investigación de la cultura en la escuela. Propone como temas de investigación las variables de todo tipo que subyacen a la cultura del profesor, del administrador y del estudiante, ya que el estudio de todas estas variables proporciona una óptica, a través de la cual los eventos en la escuela se ven de forma diferente, "por ejemplo, pueden identificarse algunos aspectos del sistema de creencias del profesor"... "compuesto de los siguientes elementos: (1) términos básicos, (2) relaciones entre los términos básicos en forma de afirmaciones de premisas básicas, y (3) relaciones entre términos y premisas en forma de afirmaciones de correlación/probabilidad, explicación causal y predicción" (Erickson, 1984, p. 55).

Para Erickson, los términos básicos de definición están relacionados unos con otros por premisas de definición y causalidad (cada alumno es un individuo; un buen estudiante es un buen trabajador, un hogar con deprivaciones es un hogar sin libros, etc.). En las proposiciones factoriales causales, las premisas "están unidas por proposiciones que relacionan personas y eventos con factores causales, probabilidad/correlación, o de predicción en la forma "si X entonces Y" "Juan es (problemático, estudiante lento, lector lento) porque procede de una familia (con padre ausente, hogar roto, deprivado background cultural)"”. Las proposiciones de correlación/probabilidad adoptan la forma de "los chicos/as problemáticos a menudo son malos lectores que o bien proceden de un hogar roto o de una familia con buen background en la que los padres exigen demasiado". Y las predic- 
ciones se resuelven, por ejemplo, como «si Juan hubiera puesto más atención, habría sido capaz de adaptarse a la clase"... "sin atención individual, los lectores lentos no serán capaces de adaptarse a la clase" (Erickson, 1984, p. 56). Nosotros entendemos que la predicción es una categoría a tener en cuenta tanto en el nivel etnográfico de los estudios en profundidad como en el estudio global de la educación en las llamadas sociedades complejas.

\subsubsection{Propuestas metodológicas}

La Antropología, hoy, desarrolla métodos y técnicas generales de investigación que son propias de las ciencias sociales. El método concreto de investigación que adopta un estudio responde lógicamente al tipo de problema que el antropólogo se propone abordar. Si se trata de un estudio urbano de carácter global, es una creencia bastante arraigada "entre los antropólogos la de que la inevitable complejidad de los datos hace que los métodos cuantitativos sean fundamentales para las investigaciones sobre las ciudades" (Wolf, Clyde y otros, 1990, p. 57). Es más, para los mismos autores, "solamente el acopio de datos sobre las poblaciones urbanas y el ulterior análisis de estos datos, dará lugar a la simplificación inherente a las teorías correctas"... "Las encuestas sociales son uno de los medios de conseguir este objetivo. Sin embargo, sus categorías de cuantificación deben establecerse mediante un análisis teórico previo. Los resultados obtenidos a partir de las encuestas pueden servir para verificar la validez general de las hipótesis construidas; ya que las encuestas se apoyan -o deberían apoyarse- en muestras elegidas de tal modo que sus resultados sean susceptibles de aplicarse a toda la población de la que se obtuvo la muestra" (Wolf, Clyde y otros 1990, p. 58). Desde la interacción entre investigación en profundidad cualitativa y cuantitativa se pueden aportar muchos datos al estudio antropológico de las sociedades avanzadas.

Por su parte, cuando se habla de antropología aplicada se está planteando la necesidad de que los antropólogos se ocupen de problemas sociales y se involucren directa e intensamente en los problemas sociales contemporáneos relevantes, a través de los llamados "experimentos de campo" en Antropología.

En la investigación científica los experimentos de campo o sobre el terreno son los que no tienen lugar en un laboratorio sino en ambientes naturales. En ellos también es necesario el control "perfecto" de la situación social de origen. Pero, en la práctica, como esto es muy difícil de conseguir, son generalmente "experimentos imperfectos". Un ejemplo de experimentos de esta clase es el que se llevó a cabo con personas que vivían en chabolas en una zona suburbial. Se les dió, sólo a algunos de ellos, viviendas de altura nuevas, para intentar comprobar qué diferencias sociales y culturales se producían y avanzar en la mejora social. Estos "experimentos imperfectos" son el tipo de experimentos que pueden llevarse a cabo en la versión aplicada de la Antropología.

Centrándonos en el campo de la Antropología de la Educación que nos ocupa, apostamos -en línea con lo anteriormente expuesto- por una Antropología de la Educación que asuma como competencias propias, partiendo de la observación descriptiva, explicativa y global, tanto la Descripción como la Explicación 
de los procesos de transmisión -adquisición de la cultura los contextos donde éstos se llevan a cabo y la interacción social que en ellos se establece-. Ciertamente, los objetivos no se agotan en la descripción ni en la explicación de lo anterior, sino que además entendemos que los datos obtenidos deben servir también para obtener un conocimiento del educando, valores, normas, etc. Apostamos aquí, así mismo, por una Antropología de la Educación que, dando un paso más, camine hacia la generalización y predicción. Una generalización, explicación y predicción reclamadas por la Antropología desde diversos ángulos, que, aun entendida con las limitaciones que señalábamos, obliga, sin duda, a ampliar el radio de acción de los instrumentos de investigación.

Por otra parte, en cuanto a su posibilidad de ciencia aplicada, de carácter más "experimental" que trataremos en un trabajo posterior, desde nuestra propuesta, la Antropología de la Educación debe intervenir sobre la realidad educativa y solucionar los problemas dentro del ámbito de su competencia, intentando avanzar en la comprensión de causa y efecto, planteándose como objetivo el diseño y elaboración de programas de mejora, cuando la situación lo requiera y sea posible.

\section{La observación en Antropología de la Educación}

Se pueden distinguir tres tipos de observación, que constituyen técnicas de investigación independiente y de distinto nivel científico: descriptiva, explicativa y global. La distinción entre ellas radica en la finalidad descriptiva, explicativa, etc., del estudio antropológico.

\section{La observación descriptiva}

En la observación descriptiva no es necesario establecer una hipótesis o grupo de hipótesis previas. Puede bastar con determinar únicamente los aspectos del campo de observacioon que van a ser objeto de atención, o, en términos más concretos, especificar las variables sobre las que se pretende obtener información. Este tipo de observación ha orientado tradicionalmente muchos estudios etnográficos. Los estudios antropológicos clásicos participan, en general, de esta modalidad y son el resultado de la convivencia con los aborígenes estudiados. En ellos, el antropólogo va tomando notas de todas las particularidades que observa en el orden social, político, cultural, económico, religioso, etc., hasta poder dar cuenta de un "estilo de vida". Ha de permanecer en el campo el suficiente tiempo como para permitir que puedan tener lugar y observarse todos los acontecimientos relevantes, a través de la observación participante, no participante, etc.

En cuanto a la observación descriptiva en Antropología de la Educación, tenemos que reconocer con Erickson que lo que hizo un clásico como Malinowski en su trabajo de campo en las islas Trobiand no puede funcionar en el caso de la escuela actual, porque sus métodos no son situacionalmente apropiados (en la escuela no se vive las 24 horas del día, ni las relaciones están influenciadas por fuertes reglas de parentesco, etc.). Algunos de los principios antropológicos gene- 
rales sobre el trabajo de campo pueden hoy servir como orientación para los etnógrafos escolares, pero no su método específico (Erickson, 1984, p. 54). Con todo, la observación descriptiva se utiliza en etnografía escolar a nivel micro y permite enriquecer el conocimiento, por ejemplo, de la interacción maestro-alumno, de la vida cotidiana del aula, etc.

Son clásicos los estudios en las escuelas americanas que han permitido entender por ejemplo, que la causa inmediata del fracaso en aprender a leer de niños pertenecientes a minorías étnicas hay que buscarla en la interacción (verbal y no verbal) entre docentes y alumnos (Ogbu, 1981, p. 29).

Dentro de la perspectiva de la observación descriptiva, George Spindler, además del referente obligado al hablar de Antropología de la Educación, representa una opción paradigmática y teórica clásica. Si bien, tiende al eclecticismo en el desarrollo de sus métodos. Pertenece a esa generación de investigadores en la que pocos podían llamarse a sí mismos antropólogos, sin haber superado el rito de ese su paso por una experiencia en el campo de las llamadas culturas primitivas. En su caso, los poblados indios de Blood y de los Menominèe de Wisconsin (G. D. Spindler y L. Spindler, 1971).

Fiel al paradigma del Funcionalismo Estructural y a la corriente Cultura $y$ Personalidad, se interesa por el registro del "fenómeno" de la transmisión cultural, el seguimiento de la norma social y la aculturación y su influencia sobre el pensamiento y la acción individuales (mental/conductual). La presencia de los supuestos teóricos de Cultura y Personalidad se hacen sentir también en su preocupación por las discontinuidades (infancia-adolescencia) que se dan en la experiencia de los individuos a medida que crecen.

La influencia de su orientación teórica en la selección y búsqueda de estrategias de recogida de datos le llevó en los años cincuenta a idear técnicas que, basadas en los tests de Rorschach, le servían para obtener información sobre los valores de los individuos o sus propias imágenes (L. Spindler y G. D. Spindler, 1958). La influencia del Funcionalismo Estructural se aprecia también en la utilización de la observación y la entrevista como medios principales de obtención de datos, ya que son la mejor forma de acceder a los comportamientos e interacciones grupales en escenarios naturales.

En cualquier caso, aunque en su orientación metodológica se observan cada vez más las exigencias científicas del diseño etnográfico, y una mayor flexibilidad y eclecticismo en los métodos, todavía conserva el enfoque tradicional artístico, ya que se entendía que la descripción de la reconstrucción cultural se hacía más creíble cuando existía sensibilidad estética (G. D. Spindler, 1970). Esta consideración científico-artística continúa todavía en algunos metodólogos, los cuales compaginan, en sus estudios antropológicos, los fundamentos artísticos con las exigencias científicas del diseño etnográfico (Kottak, 1996, p. 27).

Sin embargo, estos estudios desarrollados desde la observación descriptiva de una escuela en particular, de una región, en una localidad concreta, de un país urbanizado, nunca pueden englobar o hacer entender la escuela de ese país como un todo. Hay que tener en cuenta a la hora de tratar de llevar a cabo este tipo de investigación que, por una parte, interesa sólo a los directamente implicados en la "políti- 
ca educativa" de esa escuela (alumnos, profesores, directores, padres, etc.). Por otra parte, existe la dificultad añadida que supone el "acceso al campo", si los profesores, directores, etc, se ven amenazados o fiscalizados por la presencia del etnógrafo.

\section{La observación explicativa}

Si bien en la observación descriptiva cabe, al menos en teoría, la descripción global "holística" de un grupo, de un fenómeno social, de un pueblo o de una escuela, el fin del estudio en la observación explicativa es necesariamente más reducido en cuanto tiende a comprobar "determinadas" hipótesis formuladas previamente. Se explica menos, pero el rigor científico es muy diferente. Siguiendo a Mayntz, la observación explicativa que él denomina sistemática se distingue de la no sistemática por los procesos y características siguientes: a) Sobre la base de una teoria explicita, se aíslan conceptualmente las distintas variables a estudiar (por ejemplo, variables de comportamiento y variables que pueden determinar el mismo). b) El proceso de observación se estructura en categorías definidas con precisión, sobre las que se ordenan todas las observaciones relevantes. c) Las condiciones situacionales de partida son sometidas a control a fin de posibilitar observaciones comparables (Mayntz y otros, 1975, p. 123).

Dentro de la vertiente de la observación explicativa podemos citar a M. Hammersley representante de la nueva etnografía en contextos educativos en sociedades complejas. Hammersley pertenece a la corriente interaccionista inglesa. Es uno de los máximos representantes de la llamada etnografia reflexiva, que se inscribe dentro de un marco más genérico: la nueva etnografía. Su punto de partida es el rechazo a la oposición entre "positivismo" y "naturalismo", a la vez que apuesta por reconstruir una comunidad científico-social donde trabajos que usen diferentes métodos puedan combinarse para lograr un mejor conocimiento del mundo social.

Su propuesta es la reflexividad. La etnografía debe entenderse como un proceso reflexivo, es decir, como parte del mundo social que ella misma estudia. En este proceso de construcción social del conocimiento caben tanto la observación participante y las entrevistas, como la metodología cuantitativa, dentro de un esquema amplio que abarca distintos marcos teóricos. Sus aportaciones, si bien se desarrollan en el campo de la educación, trascienden y tienen amplia relevancia en el discurso antropológico actual. Sus tesis pueden situarse teóricamente dentro del interaccionismo simbólico y del paradigma de la definición social.

Hammersley desplegó un amplio abanico de estudios escolares en los que hace hincapié en la necesidad de partir de un diseño etnográfico previo con descripciones precisas, organización clara y dimensiones y categorías de análisis discretas. Como ejemplo, uno de sus estudios más conocidos es el estudio del rol del profesor en el aula (Hammersley, 1977 y 1979).

\section{La observación global}

Esta técnica puede considerarse, desde nuestra perspectiva fundamental para el estudio antropológico en general y para la Antropología de la Educación en par- 
ticular, dentro de las llamadas sociedades complejas (sociedades populosas con estratificación social y gobiernos centralizados), cuando se quieren obtener datos válidos y representativos de la variación de un país (estratificación social, roles, comportamiento en relación al género, etc.), con respecto a determinados tópicos de análisis. En esta técnica, la combinación de investigación con encuestas y estudios en profundidad (etnográficos) puede proporcionar nuevas perspectivas para el estudio de la cultura y la vida social de carácter general, sobre un presupuesto básico en cualquier investigación: tener un conocimiento directo (en profudidad) y global del campo de estudio, que permita la comparación.

Los estudios en profundidad constituyen la fase previa y preparatoria de la encuesta posterior. Su finalidad es comprender lo más profundamente posible el ambiente natural, económico, social, cultural y humano al que se va referir el estudio, así como las condiciones genéricas que caracterizan el universo a estudiar de cualquier tipo que sea.

En el espíritu de esta técnica subyace la idea de que las personas se ven influenciadas por las condiciones culturales y sociales en las que desarrollan sus vidas. Estas condiciones se resuelven no solo a nivel micro sino también, en gran medida, a nivel macro y se considera que estos condicionantes, en cierta medida, pueden hacer sus comportamientos posibles o incluso inevitables. De ahí la necesidad de conocer, previamente a la realización de la encuesta, los diversos intereses, actitudes, condicionantes, conflictos sociales, etc., de la población a investigar. Sin duda, nada puede reemplazar un contacto directo del observador con su campo de estudio, ninguna técnica es capaz de sugerir tantas ideas nuevas como los estudios en profundidad, pero a la vez, si éstos han de ser generalizados y aportar algo a la comprensión global de una sociedad, se necesita, además, la encuesta por muestreo. Es decir, la combinación de ambos procedimientos, que es lo que caracteriza la observación global como técnica. Al trabajar en las sociedades actuales, algunos problemas parecen propios de las situaciones culturales complejas en los que coexisten mayorías y minorías étnicas o varias subculturas, otros son comunes en cualquier situación etnográfica. Por ello, esta técnica ofrece, en el campo antropológico, grandes posibilidades.

El trabajo de campo llevado a cabo por Teresa San Román (1984) sobre los gitanos españoles es una muestra de cuanto decimos. Cuando se propuso estudiar a los gitanos, nunca se pensó en estudiarlos a todos. Aceptó que la única posibilidad era aislar una o dos comunidades y realizar el trabajo en ellas.

El trabajo de campo de esta investigación llevó a establecer ciertas hipótesis sobre la famila, vivienda, etc., en la cultura gitana y se pusieron de manifiesto las causas que se manejan como explicación de las situaciones que se vivían. Pero, en relación con las conclusiones que se tenían al terminar el trabajo etnográfico, se era consciente de que éstas no se podían extender a toda la población gitana de Madrid, ni a fortiorí a los gitanos españoles, sin haberlas comprobado. Se entendía que del "trabajo de campo" uno sale con un manojo de hipótesis científicas pero con nada más que pueda llamarse científico (San Román, 1984, p. 127). El estudio estadístico que siguió y que se realizó con muestras extraídas de las distintas situaciones en que vivían los gitanos (chabolas, viviendas de altura, unida- 
des vecinales de absorción) obligó a replantear las conclusiones para que tomaran la forma de hipótesis expresadas en categorías susceptibles de comprobación. En el tratamiento estadístico posterior se encontraron en algunos casos proporciones bajísimas para relaciones entre variables que en todo momento habían parecido evidentes en el trabajo de campo previo. Al poner a prueba (contrastación) una buena parte de las conclusiones provisionales del trabajo de campo, las más resultaron corroboradas, pero otras tuvieron que rechazarse (San Román, 1984).

Dentro de esta perspectiva de observación en nuestro campo cabe citar entre otros un reciente estudio sobre la cultura de la norma en los escolares españoles (Pérez Alonso-Geta, 1998). Donde se analizan desde distintos parámetros la transgresión y seguimiento de la norma en escolares de 8 a 14 años.

\section{Conclusión}

En el ámbito de la Antropología en general y de la Antropología de la Educación en particular, el reconocimiento de la investigación empírica como actividad dominante es bastante unánime (descripción etnográfica, observación participante, etc.). Sin embargo, en cuanto disciplina científica, la Antropología de la Educación, entendemos que según los casos ha de utilizar "también" la metodología basada en la lógica del método hipotético deductivo, que se caracteriza por la elaboración de hipôtesis explicativas y su contrastación. Desde esta óptica, el orden secuencial para acceder al análisis de los procesos (adquisicióntransmisión; mental-conductual; etc.) parte de los resultados obtenidos en determinados grupos de sujetos, enfrentados a situaciones concretas, mediante los estudios en profundidad (etnográficos). Sin embargo, estos resultados sólo nos proporcionan la visión de la situación puntual de que se trate e indicios más o menos fiables de situaciones más amplias, que, si han de ser generalizados (en sociedades complejas) o mejorados (Antropología aplicada), deben someterse a contrastación.

En Antropología de la Educación, al igual que en otras ciencias sociales, la progresión explicativa debe ir en línea con lo que se apuntaba desde la descripción pormenorizada de lo "conductual" y los acontecimientos y procesos que lo rodean, hacia las "estructuras" y los "patrones" comportamentales que nos den razón de por qué (explicación) ocurren las conductas de la manera en que lo hacen. Estas explicaciones antropológico-educativas desde nuestra perspectiva precisan, hoy, una referencia tanto a las estructuras micro (individuos, pensamiento y acción individual) como a las macro (cultura, normas y valores sociales, sistemas mundiales, etc), y a las estructuras intermedias en el "continuum" micromacro (familia, escuela, etc.). Además, necesitan estar integradas en un esquema conceptual que reúna algunas propiedades básicas, como son: a) Una delimitación clara de conceptos, que distinga claramente las categorias a estudiar. b) La influencia o poder causal de las estructuras (micro-macro) y procesos (transmisión-adquisición) sobre los fenómenos socioculturales, acontecimientos o acciones que aparecen en la observación. c) La coherencia y estabilidad de las hipótesis. 
PETRA M. PÉREZ ALONSO-GETA

ANTECEDENTES DE LA CONSTRUCCIÓN TEÓRICA EN ANTROPOLOGÍA

Si no fuera así, los hallazgos no serían útiles, convirtiendo a la ciencia en mero instrumento vacío para la predicción (Campbell, Daft y Hulin, 1982). No en vano: "La fuerza de una teoría no está en que dé cuenta de las viejas leyes, sino en su poder de predicción. En su poder para sugerir nuevas leyes que puedan ser confirmadas" (González, 1987, p. 81). Desde nuestra perspectiva, en definitiva, nada parece oponerse a que la vinculación entre las teorías y los datos se establezcan en Antropología de la Educación en las llamadas sociedades complejas de manera semejante a como se establecen en otras ciencias sociales.

\section{BiBLIOGRAFÍA.}

BARNES Y BARNES, M. S. (1896): "Education among the Aztecs". Leland Stanford Jr. University, Studies in Education. Stanford, CA.

BeATTIE, J. (1970): Otras culturas. F.C.E. México.

BENEDICT, R. (1934): Patterns of culture. Hougnton, Miffin. Boston.

Berger, P. y Luckman, T. (1968): En La construcción social de la realidad. Amorrortu. Buenos Aires.

BOAS F. (1930): "Some problems of methodology in the social sciences". En: Race, language and culture. Mcmillan. New York.

BRAMELD, T. (1961): Bases culturales de la Educación. Eudeba. Buenos Aires.

CAMPBell, J. P., DAFT, R. L. Y Hulin, C. L. (1982): What to Study. Generating and developping research questions. Sage Pub. Beverly-Hills, Los Angeles.

Chamberlain, A. (1896): "Child and Childhood in Folk Thought". Macmillan. New York.

CHOzA, J, (1985): Antropologías positivas y antropologias filosóficas. Cenlit. Navarra.

COLOM, A. Y JANER, G. (1995): "El modelo cultural en la construcción de la Antropología de la Educación". En: Cuestiones de Antropologia de la Educación. Ceac. Barcelona.

COMPTE, A. (1929): Systeme de politique positive. Tomo I. Paris.

EDDY, E. M. (1985): "Theory research and application in educational anthropology". Antbropology \& Education Quarterly, 16, pp. 82-104.

EISENHART, M. A. (1988): "The ethnographic research tradition and mathematics education research". En: Joumal for Research in mathematics education, vol. 19, 2, pp. 99-114.

ERICKSON, F. (1984): "What Makes School Ethnography "Ethnographic", En: Antbropology \& Eucation Quarterly, vol. 15, pp. 51-66.

FLETCHER, A. C. (1888). "Glimpses of child-life among the Omaha Indians". Journal of American folklore, 1, pp. 115-123.

GEERTZ (1996): La interpretación de las culturas. Gedisa. Barcelona.

GoETz. J. P. (1978): "Theoretical Approaches to the Study of Sex-Role Culture in Schools". En: Anthropology \& Education Quarterly, vol. 9, pp. 3-21.

- Y LE COMPTE, M. D. (1988): Etnografía y diseño cualitativo en investigación educativa. Morata. Madrid.

GoNZÁlez, A. (1987): La construcción teórica en Antropología. Anthropos. Barcelona.

HAMMERSLEY, M. (1977): "School learning: The cultural resources required by pupils when answering a teacher's question". En Woods, P. Y HAMMERSLEY, M. (comp.): School Experience. Croom Helm. Londres.

- (1979): "Toward a model of teacher activity". En EgGleston, J. (comps.): Teacher Decision-Making in the classroom. Rout Ledge \& Kegan Paul. Londres. 
- y Atkinson, P. (1994): Etnografía y Métodos de investigación. Paidós. Barcelona.

Howard, M. (1995): La cultura del conflicto. Las diferencias interculturales en la práctica de la violencia. Paidós. Barcelona.

JACOB, E. (1987): "Qualitative research traditions: A review". En: Review of Educational Research, 57, pp. 1-50.

KAPLAN, D. y MANNERS, R. (1988): "Antropología: viejos temas y nuevas orientaciones". En LloberA, J. R.: La Antropología como ciencia. Anagrama. Barcelona.

KотTAK, C. (1996): Antropología. Una exploración de la diversidad bumana. McGraw-Hill. Madrid.

LeVINE, R. A. (1970): "Research Design in Anthropological Field Work". En NAROLL, R. Y COHEN (eds.): A Handbook of method in cultural Antbropology. Columbia, U.P. New York, pp. 183-195.

MAYNTZ, R. Y OTROS. (1975): Introducción a los métodos en la ciencia social empírica. Alianza. Madrid.

MEAD, M. (1972): Adolescencia, sexo y cultura. Laia. Barcelona.

MERTON, R. K. (1970): Teoría y Estructuras Sociales. F.C.E. México.

MURDOCK (1974): "Muestra etnográfica mundial». En LloBERA, J. R. (comp.): La antropología como ciencia. Anagrama. Barcelona

NADEL, S. F. (1974): Fundamentos de antropología social. F.C.E. México.

NAGEL, E. (1978): La estructura de las ciencias. Problemas de la lógica de la investigación científica. Paidós. Buenos Aires.

OgBu, J. U. (1981): "School Ethnography: A Multilevel Approach". En: Anthropology \& Education Quarterly, vol. XII, 1, pp. 3-29.

Pérez Alonso-Geta, P. Ma (1998): La cultura de la norma de los escolares españoles (8-14 años). En prensa.

Popper, K. (1995a): "La autonomía de la sociología”. En Miller, D. (comp.): Popper, escritos selectos. F.C.E. México.

- (1995b): "La teoría holística de la experiencia”. En Miller, D. (comp.): Popper, escritos selectos. F.C.E. México.

RITzer, G. (1975): Sociology: A Multiple Paradigm Science. Allyn and Bacon. Boston.

- (1993): Teoría sociológica contemporánea. McGraw-Hill. Madrid.

SAN ROMÁn, T. (1984): Gitanos de Madrid y Barcelona. Ensayos sobre aculturación y etnicidad. Ediciones de la Universidad Autónoma de Barcelona. Barcelona.

SÁNCHEZ, N. (1996): “Introducción”. En GEerTz, C.: Los usos de la diversidad. Paidós. Barcelona.

SINDELL (1974): "Some discontinuities in the Enculturation of Mistassiné Cree Children". En SPINDLER, G.: Education and Cultural process: Toward an Anthropology of Education. Holt, Rinehart and Winston, pp. 331-341.

SMith, L. M. y GeOFFry (1968): The complexities of an urban classroom: An Analysis Toward a General Theory of Teaching. Holt, Rinehart and Winston. New York.

SMITH, L. M. (1974): "Reflections on Trying to Theorise from Ethnographic DATA". En: Antbropology \& Education Quarterly, vol. 5, 1, pp. 18-24.

SPINDLER, L. y SPINDler, G. D. (1958): "Male and Female adaptations in culture change". En: American Antbropologist, 60. New York, pp. 217-233.

Spindler, G. D. (1970): Being an Anthropologist: Field Work in Eleven Cultures. Holt, Rinehart, and Winston. New York.

- y SPIndere, L. (1971): Dreamers without Power: The Menomini Indians. Holt, Rinehart, and Winston. New York.

Stevenson, M. C. (1887): "Religious Life of the Zuno Child". U.S. Bureau of Stocking, George W. Jr. Washington, D.C. 
PETRA M. PÉREZ ALONSO-GETA

SuPpes, S. (1979): "La estructura de las teorías y el análisis de datos". En SupPes, F.: La estructura de las teorias científicas. Editora Nacional. Madrid.

Tindall, B. A. (1976): "Theory in the study of cultural transmission". En: Annual Review of Anthropology, 5, pp. 195-208.

URIEL, E. (1995): Análisis de datos. Series temporales y análisis multivariante. Editorial A.C. Madrid.

Wolf, E. R., Clyde, J. y otros. (1990): Antropología social de las sociedades complejas. Alianza. Madrid. 